\title{
Sachregister zu Band 74
}

Die fett gedruckten Zahlen bezeichnen Eigenarbeiten, Bb. = Buchbesprechung.

A

Ablatio retinae, Pathogenese und Therapie der -298

und ihre Behandlung 69

Verschluß der Netzhautrisse bei - durch den Thermokauter 322

Operation der -232

Goninsche Operation der - 103, 123, 184

die bisher als geheilt veröffent-lichten Fälle von - 395

Aderhaut s. Chorioidea

Affenmyopie 312

Alkaloide, toxisch-entzündlicheWir-

kungen von - auf das Auge 206 Alkoholin j ektion in eine Blut-

geschwulst mit schweren Ver-

letzungsfolgen 107 Allästhesie, optische 291 Allergische Erkrankungen 68,

197 Amaurotische Idiotie 295 Angiomatosis retinae 195 a- Anatomie der - 123 Angiospasmen, gef äßerweiternde

Medikamente bei retinalen - 403 Anisokorie, Bedeutung der - bei

homonymer Hemianopsie 278 Ankylostoma, Augenaffektionen

bei - 127 Anzeigepflicht bei gewerblichen

Augenkrankheiten 72 Argentumeinträufelung, schwere

Augenverletzung eines Neugebo-

renen durch - 107 Arteriosklerose bei Retinitis al-

buminurica 315 Auge, das weibliche und männliche

- in der Kunst 112 Augenbewegungen, die durch das

Okzipitalhirn vermittelten -- 290

- $\quad$ die bei Läsion des hinteren Längs-bündels auftretenden Störungender - 91 .

- $\quad$ Störungen der Koordination der-- bei Tabes 316

Augendruck s. Druck Auge nheilkunde 404 (Bb.) Augenhintergrund, spektroskopi-

sche Untersuchungen des - 200 Augenlider s. Lider Augenspiegeluntersuchung,

Vorteile intensiver Lichtquellen

für die - 223 - Niedervoltlampe für - im rot-

freien Licht 229

Augentropf en, Kokainismus durch

Gebrauch von --104 Augenverletzungen 107, 108 - durch Klettenhaare 205 Aussparung,

makulare 284 Avitaminosen, Beziehungen der -

zu den Augenkrankheiten 73 Azetylcholin bei retinalen Angiospasmen 403

B

Bifokalbrille, bewegliche 95

Bindehaut s. Conjunctiva 
Blepharoconjunctivitis, Behandlung der - 390

Blickkrämpfe bei Encephalitis epi-demica 75

Blinde, Beschäftigung der --71

Blinzelreflex 295

Bouillon, Berufsverbrennung der Augen durch heiße - 176

Brillen, stenopäische 117

C Chi asm a, Anatomie und Pathologie des - 269 ff. ! Chininerblindung, vorübergehen-de 50

Chorioideasarkom, sympathische

Ophthalmie bei - 146 Chorioretinitis, umschriebene mit sektorenförmigem Gesichtsfeld-ausfall 200 Chorioretinitissympathica, iso-

lierte 125, 308Conjunctiva bulbi, chronischerEntzündungsprozeß der - 306i - chronische

Lymphstauung der -j 306

Cornea, eigenartige Veränderungender - bei Heilmittelidiosynkrasíe। 205

Epitheldegeneration der - nach einer Glassplitterverletzung vor 6 Jahren 393

primäre Fettinfiltration der -183

periphere Rinnenbildung der -92

Deckung perforierender Wunden der - 101

Corpus ciliare, Melanosarkom des

í $\quad-394$

i Corpus geniculatum externum, Verhalten des - bei Blindheit 280, 281

DDakryoadenitis, Röntgenbestrah-1 lung bei akuter - 105

Sachregister

zu Band 74.

407

Denigsche Plastik bei Kalkver-

ätzung bzw. trachomatösem Pan-

nus 321 Dentale Herdinfektion, Iritis

nach - 84 Doppeltsehen, zerebrales mono-

kulares 292 Druck, Reaktion d. Augendrucks

bei trauma tischer Schädigung ei-

nes Auges am andern 125 Drusenbildung im Sehnervenkopf

bei kegelförmiger Papule 89 Durchlässigkeit der brechenden

Teile des Auges für Grenzstrah-

len 94

E Einäugigkeit, komplizierte 117 Eisensplitter in der Linse 107 Encephalitis epidemica, Blickkrämpfe bei - 75 Entoptische Erscheinungen im

monochromatischen Licht 181 Enucleatio bulbi mit Knorpel-

einpflanzung nach Magitot 116 Ernährungsstörungen des Kin-

desalters, Beziehungen der - zu

den Augenkrankheiten 73 Exophthalmus, rezidivierender

einseitiger - 82 - und Landkartenschädel 120,203

$\Gamma$

Farbenperzeption, zerebrale Stö-

rungen der - 288 Fettinfiltration, primäre - der

Hornhaut 183 Fettkörnchenzellenbef unde in 
der Markscheide des Kaninchen-

auges 54 Feuerstar als Gewerbekrankheit

114 Flimmerskotom, Verhalten des -

zur Hemianopsie 285, 286 Fortbildungszyklus für Augen-

ärzte an der Berliner Universitäts-

Augenklinik 67 Freie Arztwahl, das Unheil der -

127 Fremdkörper, retrobulbäre 108 Fütterungstuberkulose in ihrem

Einwirken auf das Auge 191

G

Gefäßerweiternde Medikamen-te beiretinalen Angiospasmen4 93

Gefäßsklerose, experimentelle 312

Gehirndruck, Stauungspapille und röntgenologische Zeichen am Schädel bei erhöhtem - 399

Gesichtsfelduntersuchung, Be-deutung der - 68

Gesichtshalluzinationen 96 Gewerbliche Augenkrankheiten und die Anzeigepflicht 72

- $\quad$ der Hydrochinonarbeiter 90Glaskörperabszeß, hervorgerufen

durch Pilzinfektion 243 Glaukom, histologischer Befund bei

- und gleichseitigem Naevus

flammeus iaciei $165-\cdot$ und Naevus flammeus 192

- $\quad$ Yibrationsmassage bei -81

- $\quad$ Zyklodialyse als augenrettendeOperation bei - mit fast auf-gehobener Vorderkammer

304

Glaukomoperationen 92 Glioblastoma multiforme 399 Glioma retinae unter dem Bilde einer

Iritis tuberculosa 35

Strahlenbehandlung beim - 113

Einfluß der Radium-Röntgenbe-strahlung bei - 81

Verlauf eines seit 12 Jahren mit Röntgenstrahlen behandelten Fal-les von - 317

Goninsche Operation der Netz-

hautablösung 103, 123, 184 Grenzstrahlen, Messungen der

Durchlässigkeit der brechenden

Teile des Auges für - 94 Großhirnangiom bei der Lindau-

schen (v. Hippelschen) Erkran-

kung 122 Grubenbildung, umschriebene --

der Papille ohne ophthalmosko-

pischen Befund 162

$\mathrm{H}$

Haftgläser, neue Anzeigen zurVer-

wendung der -189

Halluzinationen, Gesichts 96

Heilmittelidiosynkrasie mit ei-

genartigen Hornhautveränderun-

gen 205 Hemianopsie, hinasale 275 -- homonyme 278

inferior, einseitige 268

Unterscheidung infra- und supra-nukleärer - 280

Herdinfektion und Auge 69

- $\quad$ Iritis nach dentaler - 84Heterochromie, Neues über die

Fuchssche -329 
v. Hippelsche Erkr an kung, Großhirnangiom bei der - 122

Holzsplitter, nach einem Jahre entfernt 107

Hornhaut s. Cornea

Hydrochinonarbeiter, gewerbliche Augenerkrankungen der - 90

Hypertoniekrankheit und ihre Bedeutung für das Auge 71

408

Sachregister zu Band 74.

Hypophysistumor, AugenstcV $\pi$ in-gen bei -- 75, 272, 273, 274

I

Idiosynkrasie gegen Heilmittelmit

eigenartigen Hornhautverände-

rungen 205 Innere Sekretion und Auge 186 - Folgen der Störungen der - für

das Sehorgan 71 Interkalarstaphylom, Operation

bei einem - 84 Iredermie 107 Iridektomie und Linsenverletzung

376 Iris, Sarkome der - 192 Iritis nach dentaler Herdinfektion

84 Iritis tuberculosa, vorgetäuscht

durch ein Glioma retinae $3 \delta$

$\mathrm{K}$

Kalkablagerung in der Linse 208 Kalkverätzung, Denigsche Plastik

bei - 321 Kältebehandlung des Trachoms

207 Kampimetrische Methodik, Ver-

besserung der - 320 Kapillarembolien bei Krebsmeta-

stasen am Auge 362 Kapillaren, Probleme der Physio-

logie und Pathologie der - 24 Karzinom, metastatisches - 399

- ungewöhnliche Augenbefunde beimultiplen -metastasen beiderOrbitae 184, 362

Karzinose, Sehstörungen bei - 202 Katarakt, Feuerstar als Gewerbe-krankheit 114

- atypische Sonnenblumen nach

Kriegsverletzung 373

nach Thyreoidektomie 206

nichtoperative Behandlung der -

7i Kataraktoperation, Vergleichder Resultate der Altersstaroperation mit und ohne vorbereiteten

Binde-hautlappen 1 - Blutansammlung zwischen den Blättern der Linsenkapsel nach - 81

bei Keratokonus 309

Naht bei Altersstaroperation 101

- $\quad$ vor und nach der - 188 Keime, Beeinflussung augenpatho-

gener - 314 Keratitis neuroparalytica 192 Keratitis pustuliformis pro-

funda syphilitica recidivans

305 Keratokonus, Kataraktextraktion bei - 309

Klettenhaare, Verletzung des Au-

ges durch $\mathbf{\square}-205$ Kokainismus durch Gebrauch von

Augentropfen 104 Konus, eigenartiger roter - am

nasalen Papillenrand 182 Kopierstiftverletzung 81 Krankenversicherung 127 Kupíersplitter, nach

7 Jahren entfernt 107

I

Landkartenschädel und Exoph- 
thalmus 120, 203 Längsbündel, die bei Läsion des

hinteren - auftretenden Motili-

tätsstörungen 91 Lichtquellen, Vorteile intensiver

- für die Augenspiegeluntersu-chung 223

Li der, Strahlenbehandlung der Tu-moren im Bereiche der - 104

Limbusnekrose nach Schwefel-säureverätzung, Lippenschleim-hautfrühplastik bei - 83

Lindausche Erkrankung, Groß-hirnangiom bei der - 122

Linse, Eisensplitter in der - 107

- $\quad$ Kalkablagerung in der - 208Linsenlosigkeit eines Auges, Ent-

schädigung bei -326

Linsenluxation 91

Linsenverletzung und Iridektomie 376

Lipoidgranulomatose 397

Lippenschleimhautfrühplastik bei Limbusnekrose nach Schwefel-säureverätzung 83

Lochbildung, Genese der - in der Macula lutea 199

Lokalisationsophthalmoskop

85 Luftembolie, Rindenblindheit

durch arterielle - 99 Lymphangitis carcinomatosa

orbitae 362 Lymphstauung, chronische - der

Conjunctiva bulbi 306

$\mathrm{M}$

Macula, zentrale Doppelversorgung der - 284

- $\quad$ Diagnose und Differentialdia-gnose der entzündlichenund nicht-entzündlichen

Erkrankungen der

- 79

- $\quad$ Genese der Lochbildung in der -199

Maculare Aussparung 284 Markscheidenentwicklung im Kaninchenauge 54

Sachregister

zu Band 74.

409

Medikamentöse Therapie der

letzten fünf Jahre 321 Melanosarkom des Ziliarköipers

394 Meningiomedes vorderen Chiasma-

winkels 272 Meridianabweichungen der Au-

gen, Bestimmungen der - 109 Mißbildungen 80 Monochromatiscries Licht, ent-

optische Erscheinungen im - 181 Multiple Sklerose, Bedeutung der

Dauer des zentralen Skotoms für

die Diagnose der - 201 Musculus obliquus inferior,

Myektomie des - 104, 187 Myasthenie, Augenstörungen bei

- 75

Myektomie des Obliquus inferior

104, 187 Myopie, akute - nach Verletzung

dureh Hochofenschlacke 119

- $\quad$ der Affen 312

$\mathrm{N}$ 
Naevus flammeus und Glaükom 192

--m histologischer Befund bei Glaükom und gleichseitigem - faciei 165

Nebenhöhlen, klinische Beziehun-gen der -- zum Sehorgan 71

Nervocidin 313

Nervus opticus, Morphologie der Neuroglia im - 248

Schädigung des - parasellaren Ursprungs 89

-A tr op hie, früh auftretende lue-tische - 29

- der nasale Faktor in der Ätio-logie der - 401

Netzhaut s. Retina Netzhautablösung s. Ablatio

retinae Neuroglia, Morphologie der - im

N. opticus und in der Retina 248 Neuro-humorale Auslösungen

im Organismus 324 Neurologie, aus den Grenzgebieten

zwischen Ophthalmologie und -

75 Niedervoltlampe für Augenspie-

geluntersuchung im rotfreien

Licht und andere augenärztliche

Zwecke 229 Novokaininjektion, retrobulbäre

$-\quad 100$

Nystagmus, photographische Re-gistrierung des - 118

- $\quad$ optomotorischer - und Blindheit118

Nystagmus der Bergleute, zum 2000. Fall von - 115

0

Occipitalpol, direkte Läsionen des

$-\quad 285$

Operationen, Vorbereitungen zu den -73

- $\quad$ Trockensterilisation bei intraoku-laren - 394

Ophthalmia sympathica, Ana-tomie des sympathisierten Auges 196

anatomische Untersuchunge $\pi$ von Bulbi, die wegen Gefahr der - enukleiert wurden 196

bei Aderhautsarkomen 146

- $\quad$ operativ geheilter Fall von - 202Ophthalmoskop, Lokalisations-O.

Optik, physiologische 80 Optochin, Uberempfindlichkeit für

- 179

O r b i t a , Krankheiten der - 404 (Bb.) Orbitalverletzung durch Billard-stock 80

$\mathrm{P}$

Pannus, Denigsche Plastik bei tra-

chomatösem - 321 P a p i 11 e, kegelf örmige -- mit Drusen-

bildung im Sehnervenkopf 89

- umschriebene Grubenbildung der

- $\quad$ ohne ophthalmoskopischen Befund 162

PapiUenrand, eigenartiger roter

Konus am nasalen - 182 Pellagra, Augenaffektionen bei -

127 Perimeter 81 Perimetrische Methodik, Ver-

besserung der - 320 Physiologische Optik 80 Pilzinfektion als Ursache eines

Glaskörperabszesses 243 Pigmentstreif en der Netzhaut 320 Pneumokokkenerkrankung des

Auges, Serumbehandlung bei -

108, 214 Pseudosklerose, Augenbefundebei 
der - 185 Pseudosklerosenring 106 Pupillarmembran, loser Zusam-

menhang von exsudativer - mit der Linsenkapsel 180 Pupillenstarre, hemianopische -

ohne Hemianopsie 279

$\mathrm{R}$

Retina, Morphologie der Neuroglia in der - 248

- Pigmentstreifen der -320

410

Sachregister

zu Band 74.

Retina. Legalisation auf der - vom optischen Standpunkt 85

pathologische Anatomie seltener Erkrankungen der - 194

der nasale Faktor in der Ätiologie der Sehnervenatrophie durch Ar-terienkrampf in der - 401

gefäßerweiternde Medikamente bei retinalen Angiospasmen 403

Retinaatrophie, familiäre 88 Retinitis albuminurica, Arterio-

sklerose bei -315 Retinitis exsudativa, Anatomie

der - 123 RetraktionsbewegungendesBul-

bus als zerebrales Herdsymptom

3"

Rindenblindheit durch arterielle

Luftembolie 99 Röntgenaufnahmen, stereoskopi-

sche 94 Röntgenstrahlentherapie am

Auge 77

- $\quad$ bei akuter Dakryoadenitis 105Röntgenologie des Schädels 79Rumpf, Beziehungen der Motilität

des - zum Sehorgan 85

S Salvarsan, stürmische Provokation

einer bislang erscheinungsfreien

Tabes durch - 323 Sarkome der Iris $192 \mathrm{~S}$ chad el, Röntgenologie des - 79 Schiötz-X-Tonometer

319 Schüller-Christiansche Krank-

heit 120, 203 Schwef elsäureverätzung, Lip-

penschleimhaut-Frühplastik bei

Limbusnekrose nach - 83 Seelenblindheit 98, 293, 294 Sehbahn 26711. (Bericht) Sehnerv s.

Nervus opticus Sehrinde, Anatomie der - $281 \mathrm{ff}$. Sehstrahlung, Anatomie der -

$281 \mathrm{ff}$. Sella turcica, Sehnervenschädi-

gung infolge Erkrankung der -89 Serumtherapie bei Pneumokok-

kenerkrankungen des Auges 214 Sklerokeratometer 95, 403 Sklerose, experimentelle - der Ge-

fäße 312 Skotom, zentrales - bei intrakra-

niellen Tumoren 201

- $\quad$ Bedeutung der Dauer des zen-tralen - für die Diagnose dermultiplen Sklerose 201

Sonnenblumenkatarakt, atypi-sche - nach Kriegsverletzung 373

Staung spapille bei erhöhtem Him druck 399

Stenopäische Brillen 117 Stereoskopische Röntgenauf-

nahmen 94 Strahlenbehandlung der Tumoren im Bereiche der Lider 104

- $\quad$ beim Glioma retinae 113Strabismus, das Problem des -noSupercilium und Pars orbitalis palpebrae superioris 105 Sympatol 98 Syphilis, moderne Diagnose und 
Therapie der -69

$\mathrm{T}$

Tabes, Störungen der Koordination der Augenbewegungen bei - 316

- $\quad$ stürmische Provokation einer bislang erscheinungsfreien - durchSalvarsanbehandlung 323

Therapie, die medikamentöse --

der letzten fünf Jahre 321 Thyreoidektomie, Katarakt nach

- 206

Tonometer, Schiötz-X-Tonometer

319

Trachom, die Lehre vom - einst und jetzt 190, 209

Ätiologie des -101

Bekämpfungdes - mit besonderer Berücksichtigung von Erfahrun-gen aus Dortmund 43, 116

- $\quad$ Kältebehandlung des - 207Tractus opticus, Verletzung des

- 277Tränensackleiden, Behandlung

der - 76 Tränenwege, einfache Technik der

Durchspülung der - 386 Trockensterilisation bei intra-

okularen Operationen 394 Tumoren, Zentralskotom bei intra-

kraniellen - 201 Tuberkulose, Stellung der Augen

im Rahmen der allgemeinen

menschlichen - 70, 129

- $\quad$ Fütterungstbk. in ihrem Ein-wirken auf das Auge 191

Tuberkulose Augenerkrankun-gen, unspezifische, insbesondere diätetische

Allgemeinbehandlung der -310

$\mathrm{U}$

Ulcus rodens, dasWesendes-198

Ulcus serpens, Klinik und Behandlung des -301

Unfallkunde 326

U. Unfallneurose als Problem der Gegenwartsmedizin 207

Uterusschleimhaut, Verpflanzung der - in die vordere Augenkam-mer mit sichtbarem

Genitalzyklus 192 